\title{
Ginsenoside Rg3 protects heart against isoproterenol-induced myocardial infarction by activating AMPK mediated autophagy
}

\author{
Gui-Zhi Sun ${ }^{1}$, Fan-Ji Meng ${ }^{1}$, Huai-Qiu Cai ${ }^{2}$, Xue-Bo Diao ${ }^{3}$, Bo Zhang $^{1}$, Xiu-Ping Bai ${ }^{1}$ \\ ${ }^{1}$ Department of Cardiology, ${ }^{2}$ Department of Ultrasonography, The Fourth Affiliated Hospital of Harbin Medical University, Harbin 150001, China; \\ ${ }^{3}$ Department of Gynaecology and Obstetrics, The First Affiliated Hospital of Harbin Medical University, Harbin 150001, China \\ Contributions: (I) Conception and design: B Zhang, GZ Sun; (II) Administrative support: XP Bai; (III) Provision of study materials or patients: FJ \\ Meng, HQ Cai; (IV) Collection and assembly of data: XB Diao; (V) Data analysis and interpretation: B Zhang, GZ Sun; (VI) Manuscript writing: All \\ authors; (VII) Final approval of manuscript: All authors. \\ Correspondence to: Bo Zhang; Xiu-Ping Bai. Department of Cardiology, The Fourth Affiliated Hospital of Harbin Medical University, Harbin 150001, \\ China; Email: zhangbo_zobe@163.com; baixiuping@medmail.com.cn.
}

\begin{abstract}
Background: Panax ginseng is a well-known medicinal herb that is widely used in traditional Chinese medicine for treating various diseases. Ginsenoside $\operatorname{Rg} 3(\operatorname{Rg} 3)$ is thought to be one of the most important active ingredients of Panax ginseng. However, the molecular mechanism underlying the beneficial effects of $\operatorname{Rg} 3$ has been elusive.

Methods: In the mouse heart injury model induced by isoproterenol (ISO), we used brain natriuretic peptide (BNP), lactate dehydrogenase (LDH) and caspase-3 ELISA kits to test myocardium injury. To test whether $\mathrm{Rg} 3$ protects myocardial injury through AMPK mediated autophagy, we used specific AMPK inhibitor in combination with Rg3. NLRP3 inflammasome related molecules such as NLRP3, ASC and caspase-1 were measured by western-blot following $\operatorname{Rg} 3$ treatment.

Results: We found that $\mathrm{Rg} 3$ significantly reduced ISO induced myocardial injury indicated by the downregulation of serum BNP and LDH. In addition, we showed that the improvement of myocardial injury by $\operatorname{Rg} 3$ was associated with enhanced expression of autophagy related protein and activation of AMPK downstream signaling pathway.

Conclusions: We observed that inhibition of AMPK significantly reversed the myocardial protective effect of $\operatorname{Rg} 3$, which is associated with a decrease of $\operatorname{Rg} 3$ induced autophagy. These together suggested that $\operatorname{Rg} 3$ may improve myocardial injury during MI through AMPK mediated autophagy. Our study also provides important translational evidence for using $\operatorname{Rg} 3$ in treating myocardial infarction (MI).
\end{abstract}

Keywords: Ginsenoside Rg3; myocardial infarction (MI); autophagy; AMP-activated protein kinase (AMPK)

Submitted Nov 26, 2019. Accepted for publication Dec 17, 2019.

doi: $10.21037 / \mathrm{cdt} .2020 .01 .02$

View this article at: http://dx.doi.org/10.21037/cdt.2020.01.02

\section{Introduction}

Myocardial infarction (MI) is a widespread medical condition that has been the leading cause of death across different countries (1). During infarction, myocardial cells are deprived of oxygen, which results in an irreversible damage to myocardial cells. Cardiac fibrosis (a form of tissue remodeling mechanisms) happened after MI has been viewed as a crucial risk factor of heart failure (HF)
$(2,3)$. The myocardial injury during infarction is mediated by many cellular and molecular mechanisms including the impairment of mitochondria, endogenous antioxidants and lipid peroxidation (4).

Isoproterenol (ISO)-induced MI is a commonly animal model for MI (5). That can mimic many clinical and pathological features of MI in human. In this model, ISO can increase the workload of myocardial cells and raise the demand for $\mathrm{O}_{2}$, which in turn leads to relative myocardial 
hypoxia. On top of that, direct $\mathrm{O}_{2}$-wasting effect of catecholamines or their autoxidation products may result in hypotension, which further intensify the infarction (6).

Autophagy is an important compensatory mechanism process in responses cellular stress, in which proteins and damaged cellular organelles are digested by lysosomes in order to provide essential nutrients for keeping cell alive (7). Autophagy is also actively involved in various heart diseases including MI (8). During MI, energy deprivation or hypoxia may lead to the activation of AMP-activated protein kinase (AMPK), a highly conserved protein serine/threonine kinase (9). AMPK can then facilitate autophagy through the regulation of metabolic pathways. Studies have shown that AMPK protects myocardiocyte from hypoxia induced injury both in vivo and in vitro (10-12).

Panax ginseng is an important traditional Chinese herbal medicine that is widely used in treating many diseases. Ginsenoside $\operatorname{Rg} 3(\operatorname{Rg} 3)$ is the active ingredients of Panax ginseng. In recent studies, it is reported that $\mathrm{Rg} 3$ can regulate mitochondrial turn-overs (13) and autophagy (14). In present study, we proposed that $\mathrm{Rg} 3$ can improve myocardial injury induced by ISO through AMPK mediated autophagy.

\section{Methods}

\section{Animals and reagents}

Balb/c mice (6-8 w, male) were purchased from the VLAT (Vital River Laboratory Animal Technology, Beijing, China). All procedures and protocols using animal were in compliance with the AMRC (Animal Management Rule of China, Ministry of Health, document no.55, 2001). Rg3 (Meilunbio, China) was diluted in DMSO for stocking solution and further diluted at indicated concentrations in culture medium. Isoproterenol (ISO) was purchased from Sigma-Aldrich (USA).

BNP and reactive oxygen species (ROS) ELISA kits and the kit for testing LDH activity were obtained from the Jiancheng Bioengineering Company (Nanjing, China). Polyclonal rabbit anti-Mouse p-AMPK (T172), p-ACC (AP0298), LC3B (A7198), Beclin1 (A7353), and p62 (A19700) antibodies were obtained from ABclonal (Wuhan, China). Polyclonal rabbit Anti-Bax, Bcl2, $\beta$-actin and secondary antibodies were purchased from Santa Cruz Biotechnology (CA, USA).

\section{Experimental protocol for drug treatment in mice}

Male Balb/c mice were housed under standard conditions and were given rodent chow and water freely. All of the experimental procedures were performed in accordance with the Guidelines for Animal Experiments from the Committee of Medical Ethics. We induced MI model using previously described experimental procedure (15). Mice were randomly divided into 4 groups: (I) control group $(\mathrm{n}=9)$ : subcutaneous (s.c.) injection with DMSO (2 mL/kg/day, for 2 days); (II) ISO group (n=9): s.c. injection with ISO $(20 \mathrm{mg} / \mathrm{kg} /$ day, for 2 days); (III) ISO plus $\operatorname{Rg} 3$ (n=9) and (IV) ISO plus $\operatorname{Rg} 3$ and compound C (n=9): s.c. injection with $\mathrm{Rg} 3(5 \mathrm{mg} / \mathrm{kg}$, every $12 \mathrm{~h})$ or compound $\mathrm{C}(10 \mu \mathrm{mol} / \mathrm{L}$, every $12 \mathrm{~h})$ for 2 days before injection with ISO. Then, Serum was isolated from blood and used for testing BNP and LDH levels. All of the animals were sacrificed by exsanguinations.

\section{Histopathology staining}

Hearts were washed, immediately fixed with $10 \%$ NBF (neutral buffered formalin) and embedded in paraffin. We then performed $\mathrm{H} \& \mathrm{E}$ staining on these tissue sections. The images are captured by a light microscopic to monitor histopathological changes.

\section{Western blotting}

Western blotting was performed as previously described (16). We isolated protein from heart and resuspended the protein in sample buffer. The protein mixture was then denatured in boiling water for $10 \mathrm{~min}$. The protein samples were loaded in $10 \%$ SDS-PAGE in running buffer. The proteins were then transferred to a nitrocellulose membrane for 2 hours at $4{ }^{\circ} \mathrm{C}$ at $200 \mathrm{~mA}$ with a transfer buffer. Nonspecific proteins were blocked by incubating the membrane with $5 \%$ non-fat dry milk in TBS-T for 1 hour at RT. Then, the nitrocellulose membrane was incubated with the primary antibodies overnight at $4-8{ }^{\circ} \mathrm{C}$ and then with secondary antibody for 1 hour at RT. The reaction was visualized by ECL. The films were scanned and then analyzed by a NIH imaging software. Protein contents were normalized to $\beta$-actin.

\section{BNP, LDH and ROS in serum}

BNP level and ROS release in serum were determined 


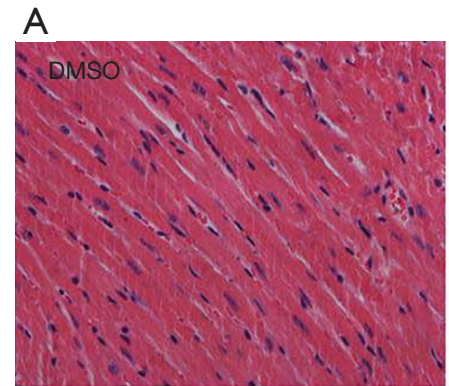

B

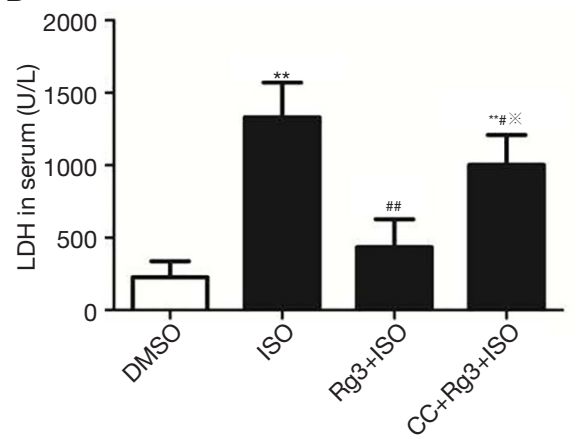

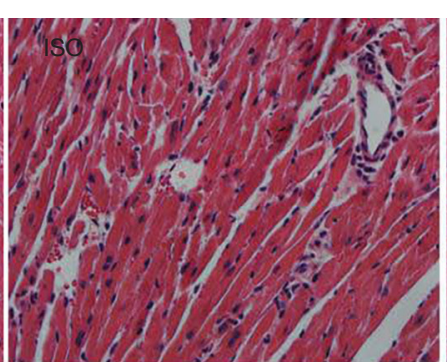
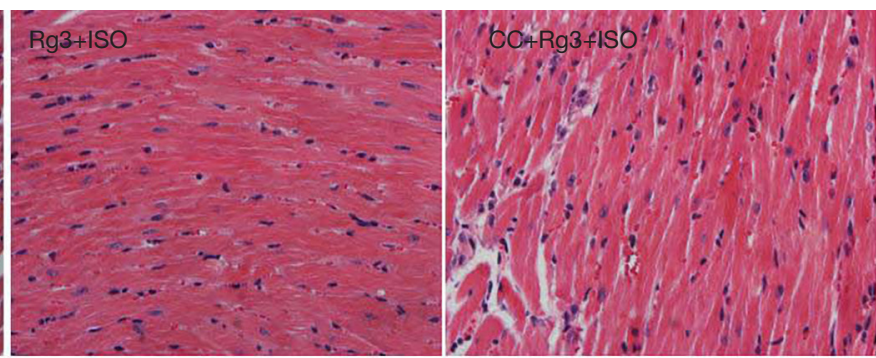

C

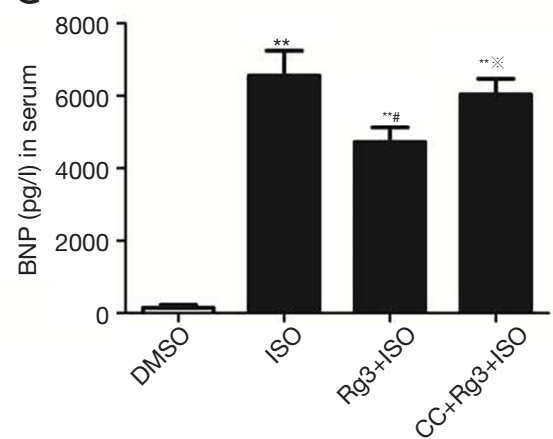

Figure $1 \mathrm{Rg} 3$ ameliorated myocardial injury in mice with ISO-induced myocardial infarction. (A) HE staining of mice heart tissue with isoproterenol-induced myocardial infarction (400x); (B) serum LDH level; (C) BNP level; (D) ROS release. Data are mean \pm SD. n=9. *, $\mathrm{P}<0.05,{ }^{* *}, \mathrm{P}<0.01$ vs. DMSO group; ${ }^{*}, \mathrm{P}<0.05,{ }^{\# \#}, \mathrm{P}<0.01$ vs. ISO group; ${ }^{*}, \mathrm{P}<0.05$ vs. Rg3 group.

by commercially available ELISA kits. ELISA plates were coated with monoclonal antibody against BNP and ROS. Dilutions of cell-free supernatants were added in duplicate, and then a second horseradish peroxidaseconjugated goat polyclonal $\mathrm{Ab}$ against $\mathrm{BNP}$ and ROS were added. After washing to remove any unbound Ab-enzyme reagent, a substrate solution was added to the wells. The color development was stopped with $2 \mathrm{~N}$ sulfuric acid, and the intensity of the color was measured at $540 \mathrm{~nm}$ on a spectrophotometer. The sensitivity of ELISA for BNP and ROS were $1 \mathrm{pg} / \mathrm{mL}$. Measurement of LDH activity in serum was as described (15), level of LDH was expressed as unit per liter for serum.

\section{Statistical analysis}

The data are represented as means \pm SDs. One-way ANOVA was used to compare three or more groups, and Tukey's post-hoc test was used to test for comparing differences between individual groups. Graphpad Prism software, version 5 , was used for data analysis and visualization. $\mathrm{P}<0.05$ was considered statistically significant.

\section{Results}

\section{Rg3 ameliorated myocardial injury in mice with isoproterenol-induced MI}

$\mathrm{H} \& \mathrm{E}$ staining of the tissue section showed that in control mice the structure of the myocardium is homogenous and clear, with no signs of edema or any inflammation. On the other hand, mice injected with ISO had moderate-tosevere necrosis of myocardium, edema, as well as massive infiltration of immune cells including both myeloid cells and lymphocytes. Furthermore, the mice treated with $\mathrm{Rg} 3$, compared to ISO-challenged mice, showed significantly improved myocardial necrosis, a substantial decrease of edema and fewer infiltration of immune cells. Interestingly, the anti-myocardium injury effect of $\mathrm{Rg} 3$ was blocked by the administration of AMPK inhibitor compound $\mathrm{C}$ (Figure 1A).

To further investigate the effects of $\operatorname{Rg} 3$ on the heart injury in vivo, we tested the serum level of LDH and BNP (two important myocardial injury markers). Rg3 significantly attenuated LDH activity by $32.7 \%$ (Figure $1 B$ ) and decreased BNP level by $72.1 \%$ (Figure 1C). We also 

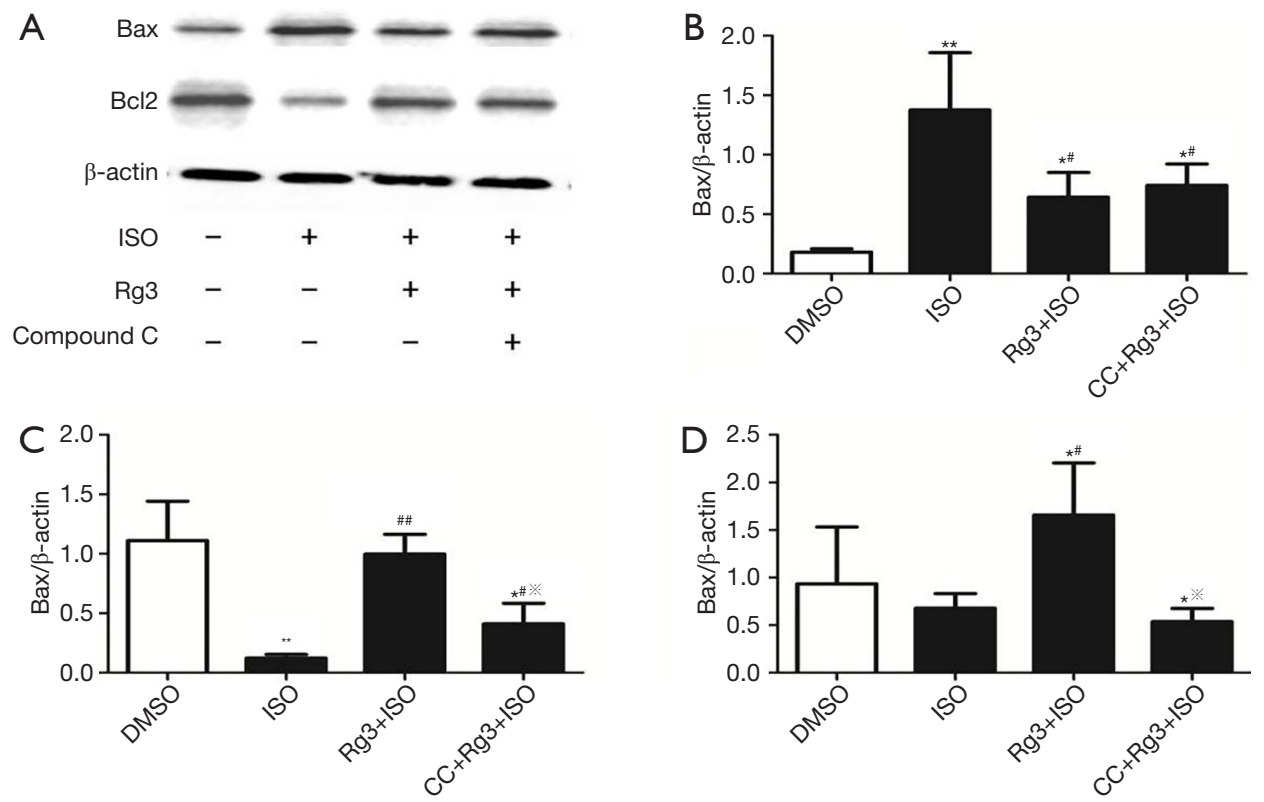

Figure $2 \mathrm{Rg} 3$ attenuated myocardial apoptosis by activating AMPK. (A) Representative protein expression of Bcl-2, Bax and $\beta$-actin; (B) quantitative analysis of protein expression of Bax; (C) quantitative analysis of protein expression of $\mathrm{Bcl}-2$; (D) the ratio of $\mathrm{Bcl}-2 / \mathrm{Bax}(\mathrm{n}=3$ ). *, $\mathrm{P}<0.05$, **, $\mathrm{P}<0.01$ vs. DMSO group; ${ }^{*}, \mathrm{P}<0.05$, \#\# $\mathrm{P}<0.01$ vs. ISO group; ${ }^{*}, \mathrm{P}<0.05$ vs. $\mathrm{Rg} 3$ group.

found $\operatorname{Rg} 3$ attenuated ROS release (Figure 1D).

\section{Rg3 treatment attenuated ISO-induced myocardial apoptosis}

We then examined the Bcl-2/Bax ratio at protein level to better understand the antiapoptotic action of $\mathrm{Rg} 3$ on myocardium. Interestingly, we found the decrease of myocardium apoptosis is associated with the reciprocal change pro-apoptotic molecule Bax and anti-apoptotic molecule Bcl-2, which has been shown to be important for myocardium survival during sepsis. These data together indicate that $\mathrm{Rg} 3$ can decrease myocardium apoptosis and improve cardiac function. Compared with ISO group, the ratio of Bcl-2/Bax strongly increased by 2.4 -fold in group treated with $\operatorname{Rg} 3+\mathrm{ISO}(\mathrm{P}<0.05$, Figure 2$)$. In addition, the cardioprotective effect of $\mathrm{Rg} 3$ was blocked by the administration of AMPK inhibitor compound C, indicating that $\mathrm{Rg} 3$ reduces ISO-induced cardiomyocyte apoptosis through the activation of AMPK (Figure 2).

\section{Rg3 promotes autophagy in ISO-induced MI models via AMPK signal pathway activation}

LC3B, p62, and Beclin-1 are commonly used markers to monitor autophagy. Using these markers, we studied the effect of $\mathrm{Rg} 3$ on autophagy during ISO-induced MI. We identified that the LC3B and Beclin-1 levels were strongly increased in $\mathrm{Rg} 3$-treated group as compared to the diseased group (ISO only, $2.2 \pm 0.29$ vs. $1.26 \pm 0.48, \mathrm{P}<0.01 ; 2.2 \pm 1.01$ vs. $0.69 \pm 0.40, \mathrm{P}<0.01$ ), while $\operatorname{Rg} 3$ treatment showed reverse effect on the p62 levels (Figure 3). These findings suggest that $\mathrm{Rg} 3$ could activate autophagy in ISO-induced MI models.

AMPK signaling pathway can enhance autophagy during multiple conditions. We also found the p-AMPK and p-ACC was increased after ISO-treated group. Pretreated with $\mathrm{Rg} 3$ abolished the increased level of p-AMPK and p-ACC. To further confirm the direct inhibitory effect of AMPK signal pathway on autophagy, we used an AMPK inhibitor, Compound $\mathrm{C}$ in the present study. Our results showed that ISO treatment decreased the levels of p-AMPK and p-ACC in myocardium, which indicates that ISO treatment reduces the activation of AMPK signal pathway. However, the Rg3 treatment reversed the inhibition effect brought by ISO $(1.85 \pm 0.27$ vs. $0.33 \pm 0.16, \mathrm{P}<0.05 ; 1.0 \pm 0.12$ vs. $0.32 \pm 0.20$, $\mathrm{P}<0.05)$, further promoting the phosphorylation of the AMPK signal pathway related proteins (Figure 4). All these results indicate that the $\mathrm{Rg} 3$ exerts heart protective function via activation of AMPK signal pathway, further promotes myocardium autophagy in ISO-induced MI models. 

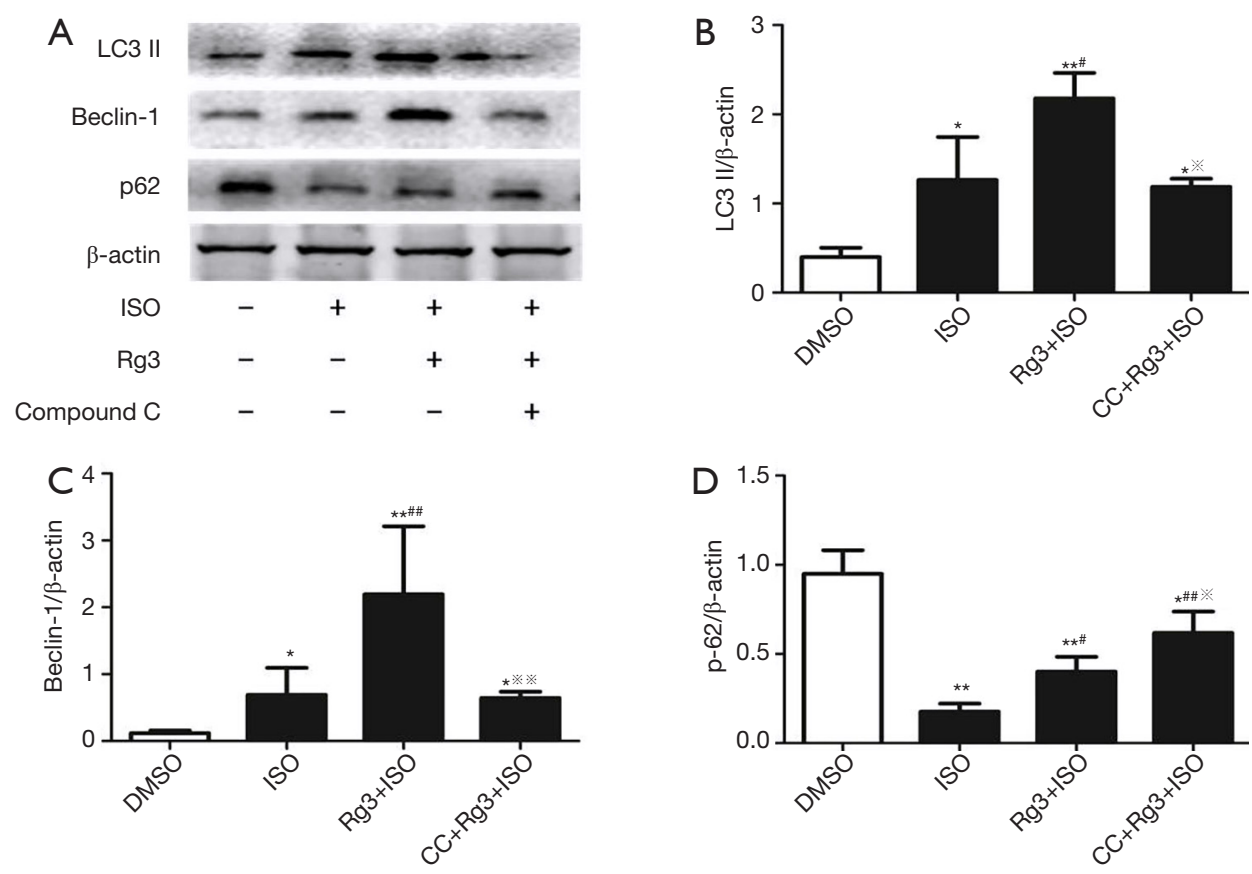

Figure $3 \mathrm{Rg} 3$ promotes myocardial autophagy by activating AMPK. (A) Representative protein expression of LC3B, Beclin-1, p-62 and $\beta$-actin; (B) quantitative analysis of protein expression of LC3B; (C) quantitative analysis of protein expression of Beclin-1; (D) quantitative analysis of protein expression of $\mathrm{p} 62(\mathrm{n}=3) .{ }^{*}, \mathrm{P}<0.05,{ }^{* *}, \mathrm{P}<0.01$ vs. DMSO group; ${ }^{*}, \mathrm{P}<0.05,{ }^{\# \#}, \mathrm{P}<0.01$ vs. ISO group; ${ }^{*}, \mathrm{P}<0.05,{ }^{* *}, \mathrm{P}<0.01$ vs. $\operatorname{Rg} 3$ group.
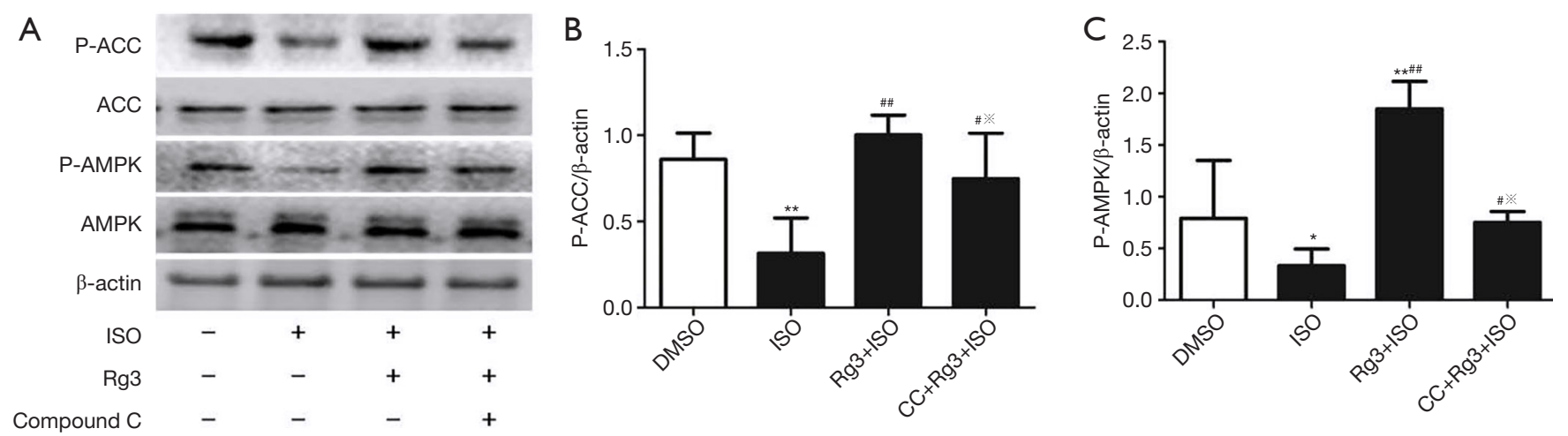

Figure $4 \mathrm{Rg} 3$ activates AMPK signal pathway with isoproterenol-induced myocardial infarction. (A) Representative protein expression of p-ACC, p-AMPK and $\beta$-actin; (B) quantitative analysis of protein expression of p-ACC; (C) quantitative analysis of protein expression of p-AMPK (n=3). *, $\mathrm{P}<0.05$, **, $\mathrm{P}<0.01$ vs. DMSO group; $, \mathrm{P}<0.05,{ }^{\# \#}, \mathrm{P}<0.01$ vs. ISO group; ${ }^{*}, \mathrm{P}<0.05$ vs. $\operatorname{Rg} 3$ group. 


\section{Discussion}

In this study, using mouse model of MI, we have demonstrated that $\mathrm{Rg} 3$ significantly attenuated ISO induced cardiac injury, as demonstrated by the reduced LDH and BNP levels in blood and reduced cardiomyocyte apoptosis. Our study also revealed that treatment of $\operatorname{Rg} 3$ upregulated mitochondrial autophagy, at least partially through AMPK signaling pathway.

MI, a serous CVD, is a prominent cause of death around the world (1). Cardiotoxicity caused by isoproterenol (ISO) at a higher dosage in an animal model exhibits a better discernment in myocardial damage and suggesting the involvement of oxidative stress. ISO is a classic tool agent in ischemic heart disease and HF investigations. ISO-induced MI animals show excessive generation of ROS followed by enhanced oxidative stress (17). Several preclinical studies have suggested that during MI, oxidative stress is significantly increased via ROS and involved in the pathogenesis of MI (18).

$\mathrm{Rg} 3$ has been demonstrated to have a broad range of beneficial effects for the cardiovascular system $(19,20)$. Our study showed that $\mathrm{Rg} 3$ exerts potential cardioprotective effects during oxidative cardiac damage as indicated by reduced levels of cardiac injury markers and ameliorated histological changes in the heart. We also observed that serum BNP and LDH activities were significantly up-regulated in the ISO-treated mice, suggesting some leakage of LDH and BNP from dying/dead cardiac cells into the circulation. In our results, mice treated with $\mathrm{Rg} 3$ had decreased necrosis with edema and infiltration of myocardium cells. This finding presents a marked improvement in myocardium necrosis, possibly due to the antioxidant effects of $\mathrm{Rg} 3$. We also found $\mathrm{Rg} 3$ treatment to ISO-challenged mice drastically reduced ROS content in myocardium.

Autophagy is a self-degradative process and is actively involved in the pathogenesis of various heart diseases (8). Autophagy is important for the quality control of mitochondria (21). In the present study, $\mathrm{Rg} 3$ was also found to have the function to remove the dysfunctional mitochondria through increasing the mitophagy in ISOinduced myocardium. Mitophagy refers to selectively degrading mitochondria by autophagy, which can reduce the accumulation of mtDNA mutations (22). When the body undergoes negative stimulations from outside world, the selective autophagy in mitochondria may occur (23). Our study found that $\operatorname{Rg} 3$ treatment has the function of inhibition of apoptosis of myocardium. Also, $\mathrm{Rg} 3$ can unregulated the autophagy-related proteins.

More interestingly, in the present study, $\mathrm{Rg} 3$ was found to promote the phosphorylation of AMPK. AMPK is an energy sensor of cells, regulating cellular energy metabolism, and its activation is conducive to rectifying metabolic disorders, making cell metabolism to be physically balanced (24). Activated AMPK can promote autophagy by inhibiting TORC1 complex (24). Therefore, we propose that Rg3 exerts mitochondrial protective function via activation of AMPK signal pathway, then further promotes mitochondrial autophagy in MI models. Furthermore, mitochondria are the key sites for fatty acid metabolism, while AMPK can affect the energy metabolism of mitochondria by influencing the activity of ACC $(24,25)$. In the current study, we also showed that $\mathrm{Rg} 3$ promoted the phosphorylation of ACC. ACC can catalyze the carboxylation of acetyl CoA and thus convert it into malonyl CoA, which is the fatty acid synthesis precursor and meanwhile inhibits the oxidation of fatty acids in mitochondria (25). AMPK can phosphorylate ACC, resulting in its decrease in activity and thus reducing the level of malonyl CoA $(24,25)$. We found $\mathrm{Rg} 3$ could significantly upregulate the phosphorylation of AMPK and ACC. In order to test the direct role of AMPK to ISOinduced cardiac dysfunction, we used AMPK inhibitor, compound $\mathrm{C}$. We found that compound $\mathrm{C}$ pretreatment could block the effects of $\mathrm{Rg} 3$ on heart protection. Which suggests that, at least partially by AMPK-autophagy signaling pathway, $\mathrm{Rg} 3$ inhibits ISO-mediated myocardium injury.

\section{Conclusions}

Taken together, our study indicates that $\mathrm{Rg} 3$ pretreatment attenuated MI induced depressed cardiac function and upregulated autophagy process through the AMPK signaling pathway. For the first time, we show that $\mathrm{Rg} 3$ protects heart against MI, at least partially through upregulation of myocardial autophagy, which may provide a deeper insight into the mechanism underlying the wide cardiovascular protective actions of $\operatorname{Rg} 3$ and provide therapeutic basis for the clinical use of $\mathrm{Rg} 3$. 


\section{Acknowledgments}

Funding: This work was supported by the Science Foundation of The fourth affiliated hospital of Harbin medical university (grant no. HYDSYJQ 201603 to B Zhang; HYDSYTB201901 to XP Bai); the Provincial Youth Science Fund of Heilongjiang, China (QC2016106 to B Zhang); the Health and Family Planning Commission of Heilongjiang Province (Grant no. 2016-119 to HQ Cai).

\section{Footnote}

Conflicts of Interest: All authors have completed the ICMJE uniform disclosure form (available at http://dx.doi. org/10.21037/cdt.2020.01.02). The authors have no conflicts of interest to declare.

Ethical Statement: The authors are accountable for all aspects of the work in ensuring that questions related to the accuracy or integrity of any part of the work are appropriately investigated and resolved.

Open Access Statement: This is an Open Access article distributed in accordance with the Creative Commons Attribution-NonCommercial-NoDerivs 4.0 International License (CC BY-NC-ND 4.0), which permits the noncommercial replication and distribution of the article with the strict proviso that no changes or edits are made and the original work is properly cited (including links to both the formal publication through the relevant DOI and the license). See: https://creativecommons.org/licenses/by-nc-nd/4.0/.

\section{References}

1. Lu L, Liu M, Sun R, et al. Myocardial Infarction: Symptoms and Treatments. Cell Biochem Biophys 2015;72:865-7.

2. Talman V, Ruskoaho H. Cardiac fibrosis in myocardial infarction-from repair and remodeling to regeneration. Cell Tissue Res 2016;365:563-81.

3. Gulati A, Jabbour A, Ismail TF, et al. Association of fibrosis with mortality and sudden cardiac death in patients with nonischemic dilated cardiomyopathy. JAMA 2013;309:896-908.

4. Basha RH, Priscilla DH. An in vivo and in vitro study on the protective effects of $\mathrm{N}$-acetylcysteine on mitochondrial dysfunction in isoproterenol treated myocardial infarcted rats. Exp Toxicol Pathol 2013;65:7-14.
5. Fan Y. Cardioprotective Effect of Rhapontigenin in Isoproterenol-Induced Myocardial Infarction in a Rat Model. Pharmacology 2019:103:291-302.

6. Cabello JB, Burls A, Emparanza JI, et al. Oxygen therapy for acute myocardial infarction. Cochrane Database Syst Rev 2013;21:CD007160.

7. White E, Mehnert JM, Chan CS. Autophagy, Metabolism, and Cancer. Clin Cancer Res 2015;21:5037-46.

8. Mialet-Perez J, Vindis C. Autophagy in health and disease: focus on the cardiovascular system. Essays Biochem 2017;61:721-32.

9. Beauloye C, Bertrand L, Horman S, et al. AMPK activation, a preventive therapeutic target in the transition from cardiac injury to heart failure. Cardiovasc Res 2011;90:224-33.

10. Tao J, Zhu W, Li Y, et al. Apelin-13 protects the heart against ischemia-reperfusion injury through inhibition of ER-dependent apoptotic pathways in a timedependent fashion. Am J Physiol Heart Circ Physiol 2011;301:H1471-86.

11. Terai K, Hiramoto Y, Masaki M, et al. AMP-activated protein kinase protects cardiomyocytes against hypoxic injury through attenuation of endoplasmic reticulum stress. Mol Cell Biol 2005;25:9554-75.

12. Yeh CH, Chen TP, Wang YC, et al. AMP-activated protein kinase activation during cardioplegia-induced hypoxia/reoxygenation injury attenuates cardiomyocytic apoptosis via reduction of endoplasmic reticulum stress. Mediators Inflamm 2010;2010:130636.

13. Sun M, Huang C, Wang C, et al. Ginsenoside Rg3 improves cardiac mitochondrial population quality: mimetic exercise training. Biochem Biophys Res Commun 2013;441:169-74.

14. Zheng X, Chen W, Hou H, et al. Ginsenoside 20(S)-Rg3 induced autophagy to inhibit migration and invasion of ovarian cancer. Biomed Pharmacother 2017;85:620-6.

15. Zhang GG, Cai HQ, Li YH, et al. Ghrelin protects heart against ERS-induced injury and apoptosis by activating AMP-activated protein kinase. Peptides 2013;48:156-65.

16. Zhang B, Liu Y, Zhang JS, et al. Cortistatin protects myocardium from endoplasmic reticulum stress induced apoptosis during sepsis. Mol Cell Endocrinol 2015;406:40-8.

17. Shahzad S, Mateen S, Mubeena Mariyath PM, et al. Protective effect of syringaldehyde on biomolecular oxidation, inflammation and histopathological alterations in isoproterenol induced cardiotoxicity in rats. Biomed Pharmacother 2018;108:625-33. 
18. Zhou SX, Zhou Y, Zhang YL,et al. Antioxidant probucol attenuates myocardial oxidative stress and collagen expressions in post-myocardial infarction rats. J Cardiovasc Pharmacol 2009;54:154-62.

19. Shi Y, Han B, Yu X, et al. Ginsenoside Rb3 ameliorates myocardial ischemia-reperfusion injury in rats. Pharm Biol 2011;49:900-6.

20. Peng L, Sun S, Xie LH, et al. Ginsenoside Re: pharmacological effects on cardiovascular system. Cardiovasc Ther 2012;30:e183-8.

21. Filomeni G, De Zio D, Cecconi F. Oxidative stress and autophagy: the clash between damage and metabolic needs. Cell Death Differ 2015;22:377-88.

Cite this article as: Sun GZ, Meng FJ, Cai HQ, Diao XB, Zhang B, Bai XP. Ginsenoside $\operatorname{Rg} 3$ protects heart against isoproterenol-induced myocardial infarction by activating AMPK mediated autophagy. Cardiovasc Diagn Ther 2020;10(2):153-160. doi: 10.21037/cdt.2020.01.02
22. Lee J, Giordano S, Zhang J. Autophagy, mitochondria and oxidative stress: cross-talk and redox signaling. Biochem J 2012;441:523-40.

23. Ding WX, Yin XM. Mitophagy: mechanisms, pathophysiological roles, and analysis. Biol Chem 2012;393:547-64.

24. Mihaylova MM, Shaw RJ. The AMPK signaling pathway coordinates cell growth, autophagy and metabolism. Nat Cell Biol 2011;13:1016-23.

25. Marcinko K, Steinberg GR. The role of AMPK in controlling metabolism and mitochondrial biogenesis during exercise. Exp Physiol 2014;99:1581-5. 